\title{
Lack of efficacy of 9-aminocamptothecin in adults with newly diagnosed glioblastoma multiforme and recurrent high-grade astrocytoma ${ }^{1}$
}

\author{
Fred Hochberg, ${ }^{2}$ Stuart A. Grossman, Tom Mikkelsen, Michael Glantz, Joy D. Fisher, \\ and Steven Piantadosi, for the NABTT CNS Consortium
}

The NABTT Central Operations Office, The Johns Hopkins Oncology Center, Baltimore, MD 21287 (J.D.F.);

The Johns Hopkins University, Baltimore, MD 21287 (S.A.G., S.P.); Henry Ford Hospital, Detroit, MI 48202 (T.M.);

Massachusetts General Hospital, Boston, MA 02114 (F.H.); and Brown University, Providence, RI 02903 (M. G.)

9-Aminocamptothecin (9-AC) was administered as a 72-h i.v. infusion every 2 weeks to a total of 99 adults with high-grade astrocytomas. Fifty-one patients with newly diagnosed glioblastoma multiforme received 9-AC treatment prior to radiation therapy and 48 patients with highgrade astrocytomas were treated at the time of tumor recurrence. Upon entrance into these research protocols, all patients had measurable disease that was evaluated on a monthly basis with volumetric CT or MRI scans. A partial response was defined by $\geq 50 \%$ reduction in the contrast enhancing volume on stable or decreasing doses of glucocorticoids. The study specified that all apparent responders would have central review of their radiologic studies and histopathology. The initial patients treated with 9-AC were also receiving anticonvulsants and were noted to have minimal myelosuppression with this chemotherapy. Thus, 9-AC doses were escalated from the previously reported maximum tolerated dose (MTD) of $850 \mu \mathrm{g} / \mathrm{m}^{2} / 24 \mathrm{~h}$. We then established new MTDs for patients receiving enzyme-inducing anticonvulsants. We

Received 20 April 1999, accepted 9 August 1999.

${ }^{1}$ Supported by NCI (CTEP) U01CA6406-07.

${ }^{2}$ Address correspondence and reprint requests to Fred Hochberg, M.D., c/o The NABTT Central Operations Office, The Johns Hopkins Oncology Center, Room 129, 600 North Wolfe St., Baltimore, MD 21287.

${ }^{3}$ Abbreviations used are as follows: 9-AC, 9-amino-20(S)-camptothecin; GBM, glioblastoma multiforme; HGA, high-grade astrocytoma; MTD, maximum tolerated dose; NABTT, The New Approaches to Brain Tumor Therapy [CNS Consortium]. defined these MTDs to be $1776 \mu \mathrm{g} / \mathrm{m}^{2} / 24 \mathrm{~h}$ for newly diagnosed, previously untreated patients and 1611 $\mu \mathrm{g} / \mathrm{m}^{2} / 24 \mathrm{~h}$ for patients with recurrent disease. Twentytwo patients with newly diagnosed glioblastoma multiforme received 9-AC at doses $\geq 1776 \mu \mathrm{g} / \mathrm{m}^{2} / 24 \mathrm{~h}$. Of these, 18 had evaluable disease on central review, and 0 of $18(0 \%)$ demonstrated a partial or complete response. Twenty-one patients with recurrent high-grade astrocytomas were treated at $1611 \mu \mathrm{g} / \mathrm{m}^{2} / 24 \mathrm{~h} ; 20$ had evaluable disease and 0 of $20(0 \%)$ had a partial or complete response. Thus, the overall response rate in the 38 evaluable patients treated at the MTD was 0 of $38(0 \%)$. Furthermore, of the 51 evaluable patients who were treated at doses less than the MTD, only one partial response was observed, yielding an overall response rate of $2 \%$. Evidence of drug failure was rapid with tumor progression in one-half of patients after 2 drug cycles. 9-AC lacks evidence of substantial activity in patients with newly diagnosed or recurrent high-grade astrocytomas. Neuro-Oncology 2, 29-33, 2000 (Posted to NeuroOncology [serial online], Doc. 99-16, December 14, 1999. URL <neuro-oncology.mc.duke.edu>)

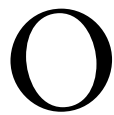
ne half of the 15,000 primary brain tumors diagnosed each year in the United States are anaplastic astrocytomas or GBMs. ${ }^{3}$ Particularly among the elderly, the tumor is increasing in incidence without concomitant improved survival (Bahemuka et al., 1988). Although patients with anaplastic or malignant oligoastrocytomas (Kim et al., 1996) may respond to procarbazine, 1-(2-chloroethyl)-3-cyclohexyl-1-nitrosou rea (CCNU), and vincristine (PCV), this treatment is without 
significant benefit in patients with glioblastomas that lack oligodendroglial elements. Clinical trials are underway to screen new drugs, such as 9-AC, for activity in HGAs (Grossman et al., 1998a).

9-AC is a member of a class of compounds that inhibit topoisomerase I (Horowitz et al., 1973; Liu et al., 1989). Included in this group is camptothecin, an alkaloid extracted from the Chinese plant Camptotheca acuminata, which inhibits in vitro and in vivo RNA and DNA synthesis in cell lines derived from tumors (Muggia et al., 1972). Phase I and II trials conducted in the 1970s using the camptothecin salt demonstrated minimal antitumor activity and significant bladder toxicity. There was renewed interest in these compounds when it became apparent that the inhibition of DNA topoisomerases plays a role in cytotoxicity. Topoisomerase I relaxes supercoiled DNA by incision and is necessary for DNA and RNA synthesis (Liu et al., 1989). Re-sealing of DNA is prevented by the binding of camptothecin to the DNAenzyme complex, resulting in the accumulation of reversible enzyme-DNA cleavable complexes (Hertzberg et al., 1989; Hsiang et al., 1985). The pharmacology of 9-AC has been studied extensively. It interacts with topoisomerase I, primarily through the lactone derivative. The substitutions at the 9 position of the A ring improve drug solubility and account for the activity of 9-AC against many solid tumor cell lines in athymic animals (Giovanella et al., 1989; Pantazis et al., 1992; Potmesil et al., 1991, 1993). Different infusion schedules have also been explored, with a 72-h infusion appearing promising (Dahut et al., 1994; Eder et al., 1998; Rubin et al., 1994; Takimoto et al., 1997). Steady-state concentrations following a 72-h continuous infusion are achieved within 48-72 h (Burris et al., 1992; Takimoto et al., 1994). Plasma lactone, postinfusion, declined in a biphasic fashion with an apparent mean terminal elimination half-life of $9 \mathrm{~h}$. Although there are some anecdotal reports of responses following the use of other members of this drug class, clinical studies of 9-AC in patients with HGAs are lacking.

We have recently reported the unexpected need to use higher doses of 9-AC in patients on enzyme-inducing anticonvulsants. (Grossman et al., 1998a; Piantadosi et al., 1998). In these studies, myelosuppression did not occur in anticonvulsant recipients receiving the MTD, but was seen in patients who received the same dose but were not on anticonvulsants. As a result, 9-AC doses were escalated from the previously defined MTD of 850 $\mu \mathrm{g} / \mathrm{m}^{2} / 24 \mathrm{~h}$ to establish new MTDs for patients receiving enzyme-inducing anticonvulsants. We defined these MTDs to be $1776 \mu \mathrm{gg} / \mathrm{m}^{2} / 24 \mathrm{~h}$ for newly diagnosed previously untreated patients with GBM and $1611 \mu \mathrm{g} / \mathrm{m}^{2} / 24 \mathrm{~h}$ for patients with recurrent HGA. In this manuscript, we present data on the efficacy of 9-AC administered at the MTD to these two populations.

\section{Methods}

This study was conducted by the NABTT CNS Consortium funded by the National Institutes of Health. The institutions participating in this research included Brown
University, Columbia University, Henry Ford Hospital, Johns Hopkins University, Massachusetts General Hospital, Moffitt Cancer Center, Northwestern University, and Wake Forest University. The clinical research studies described in this manuscript were approved by the National Cancer Institute's Center for Trial Evaluation Program and by the Institutional Review Boards at each participating institution.

\section{Patient Population}

Two clinical research protocols were conducted concurrently to determine the response rate of 9-AC given at the MTD in patients with HGA. The results are combined for purposes of this manuscript. The first protocol was for patients with newly diagnosed supratentorial GBM who had received no prior radiation or chemotherapy. The patient eligibility criteria were as follows: age $\geq 18$ years; able to provide consent; no prior or concurrent malignancy or hemorrhagic cystitis; histologically confirmed supratentorial grade IV astrocytoma (GBM); no prior irradiation, chemotherapy, biologic or hormonal agents for the brain tumor; postoperative Karnofsky performance $\geq 60$; residual measurable contrast-enhancing tumor on MRI or CT scan; and normal hematologic (absolute neutrophil count $\geq 1500 / \mu 1$, platelets $\geq 100,000 / \mu \mathrm{l}$ ), renal, and hepatic (creatinine $\leq 1.7 \mathrm{mg} / \mathrm{dl}$, total bilirubin $\leq 1.5 \mathrm{mg} / \mathrm{dl}$, transaminase $\leq 4$ times above the upper limits of the institutional norm) function. On this protocol, 9-AC therapy, initiated usually within three weeks of histologic confirmation, was planned to a maximum of six cycles. Standard radiation therapy was administered after completion of the 9-AC or upon radiographic progression of tumor.

The second protocol was for patients with recurrent or progressive HGAs. The patient eligibility criteria were identical to those described above, with the following additional requirements: recurrent or progressive, measurable disease following radiation therapy; eligible histology of GBM or anaplastic astrocytoma; estimated life expectancy of $\geq 2$ months. Patients who had received another topoisomerase inhibitor or who had received more than one prior chemotherapy regimen were excluded from participation. Patients received 9-AC until there was evidence of tumor progression or unacceptable toxicity. When either of these occurred, they were taken off the study.

\section{Statistical Considerations}

Both studies used a design incorporating an initial phase I study with escalating dose and the continual reassessment method, followed by a two-stage phase II study. The phase I study commenced at 1260 or $850, \mu \mathrm{g} / \mathrm{m}^{2} / 24 \mathrm{~h}$ for anticonvulsant recipients or nonrecipients, respectively. Three patients were treated at each dose level until the MTD was reached. The phase II studies were designed to detect a $20 \%$ improvement over a background response rate of $10 \%$. Eighteen patients were treated in the first stage. If two or fewer patients showed response, advancement to phase II was stopped; otherwise 17 additional patients were treated (35 total). In addition, both phase II 
studies had the secondary objective of estimating diseasefree progression, disease-free survival, and frequency of serious or life-threatening toxicities. All patients were evaluable for response unless appropriate scans were not obtained.

\section{Drug Administration}

The study drug, unstable in the presence of plastic, was diluted in $1 \mathrm{ml}$ of dimethylacetamide $(5 \mathrm{mg} / \mathrm{ml})$ and rediluted in special diluent containing polyethylene glycol and phosphoric acid buffer to a concentration of 100 $\mu \mathrm{g} / \mathrm{ml}$. This was placed in a Pharmacia CADD pump with a special cassette that provides a stable environment for the 9-AC. Chemotherapy was given for $72 \mathrm{~h}$ as a continuous i.v. infusion to these outpatients. Anti-emetics were provided as required.

\section{Response Assessment}

Volumetric MRI or CT scans were performed once each month. The following definitions were used for purposes of this study: complete response, disappearance of all contrast-enhancing tumor in the absence of glucocorticoids with stable or improving neurologic function; partial response, $\geq 50 \%$ reduction in contrast-enhancing tumor volume on a stable or decreasing dose of glucocorticoids with stable or improving neurologic function; progressive disease, $\geq 25 \%$ increase in contrast-enhancing tumor volume or the development of new lesions or progressive neurologic dysfunction; and stable disease, failure to meet the criteria for partial response or progressive disease. All serial MRI and CT scans for all patients responding to treatment at the MTD were reviewed centrally by the NABTT Central Operations Office (Tom Mikkelsen, M.D.). In addition, the pathology of all responding patients was reviewed at the NABTT Central Operations Office (Peter Burger, M.D.).

\section{Results}

\section{Demographic Data}

The demographic data on the evaluable patients treated at or above the MTD are seen in Table 1.

\section{Patients Treated Below the MTD}

Fifty-one evaluable patients were enrolled in the phase I dose escalation portions of these studies and received doses below the finally determined MTD of 9-AC. Only one of these patients had a partial response.

\section{Patients Treated at or Above the MTD}

Forty-three patients received $9-\mathrm{AC}$ as a 72 -h continuous infusion at or above the MTD. After central review of their radiological studies, 38 were judged to be evaluable for response. Twenty-two patients (18 evaluable) with newly diagnosed GBM received 9-AC before radiation and 21 (20 evaluable) with HGA (11 with GBM and 9 with anaplastic astrocytoma) received 9-AC at the time of tumor recurrence. All patients treated at recurrence had received radiation $>5000 \mathrm{cGy} ; 13$ had received one prior chemotherapy regimen; and 3 were treated for a malignant recurrence of a prior low-grade astrocytoma or oligodendroglioma.

At the MTD, 9-AC was associated with considerable myelosuppression (Table 2). Forty-nine percent of the 38 evaluable patients required dose reductions for myelosuppression. This figure rose to $63 \%$ in the setting of prior radiation or chemotherapy. Grade 3 or 4 neutropenia was noted in $78 \%$ of all patients, including $67 \%$ of the newly diagnosed patients and $89 \%$ of the recurrent patients. In addition, Grade 3 or 4 thrombocytopenia was noted in $32 \%$ of these evaluable patients, including $17 \%$ of the newly diagnosed patients and $47 \%$ of the recurrent patients.

There were no complete or partial responders in the 18 evaluable patients with newly diagnosed GBM treated before irradiation with 9-AC at the MTD dose $(\geq 1776$ $\mathrm{\mu g} / \mathrm{m}^{2} / 24 \mathrm{~h}$ for $72 \mathrm{~h}$ every 2 weeks). In addition, no complete or partial responders were noted in the 20 evaluable patients with recurrent HGA treated at the MTD dose $\left(\geq 1611 \mu \mathrm{g} / \mathrm{m}^{2} / 24 \mathrm{~h}\right.$ for $\left.72 \mathrm{~h}\right)$. Thus, the overall response rate in these 38 evaluable patients treated at the MTD was $0 \%$ (Table 3 ). The upper $95 \%$ (one-sided) confidence found on 0 out of 38 is 0.08 . Combining those treated with 9-AC at any dose, only one of 89 patients $(1 \%)$ with an HGA had a partial response to this agent.

Furthermore, tumor progression on 9-AC was rapid with $>50 \%$ of patients progressing after fewer

Table 1. Demographic information on evaluable patients

\begin{tabular}{lccc} 
Parameter & Total patients $(n=38)$ & Newly diagnosed GBM $(n=18)$ & Recurrent HGA $(n=20)$ \\
\hline Median age in years (range) & $50(26-75)$ & $53(26-65)$ & $46(28-75)$ \\
\% Male & 76 & 83 & 70 \\
\% Receiving prior radiotherapy (>5000 cGy) & 53 & 0 & 100 \\
\% Receiving one prior chemotherapy regimen & 34 & & 65 \\
Histology & & 100 & 55 \\
$\%$ GBM & 76 & NA & 45 \\
$\%$ Anaplastic astrocytoma & 24 & NA & 15 \\
\% Prior low-grade tumors & b & 8 &
\end{tabular}

Abbreviations: GBM, glioblastoma multiforme; HGA, high-grade astrocytoma; NA, not applicable.

${ }^{a}$ Excluded are anaplastic oligodendroglioma because none were seen.

${ }^{\text {b}}$ Three patients (8\%) were initially diagnosed with low-grade astrocytoma and subsequently progressed to a high grade. 
Table 2. Myelosuppression in evaluable patients

\begin{tabular}{|c|c|c|c|}
\hline Indicator & Total patients $(\%)(n=37)$ & Newly diagnosed GBM (\%) $(n=18)$ & Recurrent HGA (\%) $(n=19)$ \\
\hline \multicolumn{4}{|l|}{ Neutropenia } \\
\hline Grade 3 & 27 & 17 & 26 \\
\hline Grade 4 & 57 & 50 & 63 \\
\hline Grade $3+4$ & 78 (32 cycles) & 67 (13 cycles) & 89 (19 cycles) \\
\hline \multicolumn{4}{|l|}{ Thrombocytopenia } \\
\hline Grade 3 & 24 & 17 & 32 \\
\hline Grade 4 & 8 & 0 & 16 \\
\hline Grade $3+4$ & 32 (12 cycles) & 17 (3 cycles) & 47 (9 cycles) \\
\hline $\begin{array}{l}\text { Required dose reduction for } \\
\text { myelosuppression }\end{array}$ & 49 & 33 & 63 \\
\hline
\end{tabular}

Abbreviations: GBM, glioblastoma multiforme; HGA, high-grade astrocytoma.

Table 3. Lack of efficacy in evaluable patients

Progression

Total patients $(n=38)$

Newly diagnosed GBM $(n=18)$

Recurrent HGA $(n=20)$

Median time to progression (days)

50

63

40

Abbreviations: GBM, glioblastoma multiforme; HGA, high-grade astrocytoma.

No complete or partial responses were seen in any of the patients.

than two infusions (1 month). The median time to progression for patients with GBM treated before irradiation was 63 days. The 20 patients with recurrent HGAs who progressed had a median progression time of 40 days. Median survival for newly diagnosed patients was 267 days \pm 239 (range $=52-1192$ days). For recurrent patients, survival was 157 days \pm 290 (range $=$ 20-1270 days).

\section{Discussion}

HGAs in adults remain incurable with surgery, radiation therapy, and chemotherapy. As the efficacy of currently available chemotherapy for these tumors is very limited, screening for active new agents is a high priority. As a result, the NABTT CNS Consortium is studying novel agents in patients with newly diagnosed and recurrent HGAs with novel agents (Grossman et al., 1998b). This approach is designed to determine rapidly whether an agent has activity warranting further study. A recently conducted study of paclitaxel in the same population revealed a striking drug interaction between the anticonvulsants and this chemotherapeutic agent. The commonly used anticonvulsants appear to induce enzymes in the hepatic P450 system and accelerate the metabolism of paclitaxel (Fetell et al., 1997). It was not anticipated that this would also occur with 9-AC, as this agent is not known to be hepatically metabolized. However, as noted above, a significantly higher dose of 9-AC is required in patients receiving enzyme-inducing anticonvulsants to achieve similar blood levels and toxicity profiles. As a result of this finding and the need to conduct dose-finding and then formal efficacy studies at the new MTD, we have considerable information about the efficacy of this agent in newly diagnosed GBM and recurrent HGA.

The results of this study clearly indicate that 9-AC does not have significant efficacy against these tumors. Eighteen patients with newly diagnosed GBMs and 20 patients with recurrent gliomas received 9-AC at the newly defined MTD using a 72-h continuous infusion schedule every two weeks. That these patients truly received an MTD is evidenced by the extent of observed myelosuppression. Serial contrast-enhancing "tumor" volumes were used to determine response. None of the 38 evaluable patients had a partial or complete response to $9-\mathrm{AC}$, and the median time to progression was $<60$ days. Furthermore, 18 of these patients were previously untreated with radiation or chemotherapy. If this agent fails to provide meaningful response in these patients, it is unlikely to work in the recurrent disease setting. These data strongly suggest that systemically administered 9AC does not warrant further study in this patient population. It remains to be seen if other members of the camptothecin family will be beneficial in the treatment of astrocytic neoplasms.

\section{References}

Bahemuka, M., Massey, E.W., and Schoenberg, B.S. (1988) International mortality from primary nervous system neoplasms: Distributions and trends. Int. J. Epidem. 17, 33-38.

Burris, H.A., Rothenberg, M.L., Kuhn, J.G., and Von Hoff, D.D. (1992) Clinical trails with topoisomerase I inhibitors. Semin. Oncol. 19, 663-669.
Dahut, W., Brillhart N., Takimoto, C., Allegra, C., Hamilton, J.M., Sorenson, J.M., Arbuck, S., Chen, A., and Grem, J. (1994) A Phase I trial of 9-aminocamptothecin (9-AC) in adult patients with solid tumors. Proc. Am. Soc. Clin. Oncol. 13, 138. (Abstract)

Eder, J.P, Jr., Supko, J.G., Lynch, T., Bryant, M., Vosburgh, E., Shulman, L.N., 
Xu, G., and Kufe, D.W. (1998) Phase I trial of the colloidal dispersion formulation of 9-amino-20 (S)-camptothecin administered as a 72-hour continuous intravenous infusion. Clin. Cancer Res. 4, 317-324.

Fetell, M.R., Grossman, S.A., Fisher, J.D., Erlanger, B., Rowinsky, E., Stockel, J., and Piantadosi, S. (1997) Preirradation paclitaxel in glioblastoma multiforme: Efficacy, pharmacology, and drug interactions. J. Clin. Oncol. 15, 3121-3128.

Giovanella, B.C., Stehlin, J.S., Wall, M.E., Wani, M.C., Nicholas, A.W, Liu, L.F., Silber, R., and Potmesil, M. (1989) DNA topoisomerase I-targeted chemotherapy of human colon cancer in xenografts. Science (Washington) 246 , 1046-1048.

Grossman, S.A, Brem, H., Piantadosi, S., and Fisher, J. (1998) The NABTT CNS Consortium: Organization, objectives and progress. Cancer Control 5 107-114.

Grossman, S.A., Hochberg, F., Fisher, J.D., Chen, T.L., Kim, L., Gregory, R., Grochow, L.B., and Piantadosi, S. (1998) Increased 9-aminocamptothecin dose requirements in patients on anticonvulsants. NABTT CNS Consortium. The New Approaches to Brain Tumor Therapy. Cancer Chemother. Pharmacol. 42, 118-126.

Hertzberg, R.P., Caranfa, M.J., and Hecht, S.M. (1989) On the mechanism of topoisomerase I inhibition by camptothecin: Evidence for binding to an enzyme-DNA complex. Biochemistry 28, 4629-4638.

Hsiang, Y.H, Hertzberg, R., Hecht, S., and Liu, L.F. (1985) Camptothecin induces protein-linked DNA breaks via mammalian DNA topoisomerase I. J Biol. Chem. 260, 14873-14878.

Horowitz, S.B., and Horowitz, M.S. (1973) Effects of camptothecin on the breakage and repair of DNA during the cell cycle. Cancer Res. 33:2834-2836.

Kim, L., Hochberg F.H., Thornton, A.F., Harsh, G.R. IV, Patel, H., Finkelstein, D., and Louis, D.N. (1996) Procarbazine, lomustine, and vincristine chemotherapy for grade III and grade IV oligoastrocytomas. J. Neurosurg. 85, 602-607.

Liu, L.F. (1989) DNA topoisomerase poisons as antitumor drugs. Annu. Rev. Biochem. 58, 351- 375 .
Muggia, F.M., Creaven, P.J., Hansen, H.H., Cohen, M.H., and Selawry, O.S. (1972) Phase I trial of weekly and daily treatment with camptothecin (NSC-100880): Correlation with preclinical studies. Cancer Chemother. Rep. (Pt. 1) 56, 515-521.

Pantazis, P., Hinz, H.R, Mendoza, J.T., Kozielski, A.J., Williams, L.J., Jr., Stehlin, J.S., Jr., and Giovanella, B.C. (1992) Complete inhibition of growth followed by death of human malignant melanoma cells in vitro and regression of human melanoma xenografts immunodeficient mice induced by camptothecins. Cancer Res. 52, 3980-3987.

Piantadosi, S., Fisher, J.D., and Grossman, S.A. (1998) Practical implementation of a modified continual reassessment method for dose-finding trials. Cancer Chemother. Pharmacol. 41, 429-436.

Potmesil, M., Giovanella, B.C., and Lui, L.F. (1991) Preclinical studies of DNA topoisomerase I- targeted 9-amino and 10, 11-methylenedioxycamptothecin. In: Potmesil, M., and Kohn, K.W. (Eds.), DNA Topoisomerases in Cancer. New York: Oxford University Press. pp. 283-298.

Potmesil, M., Giovanella, B.C., Wall, M.E. (1993) Preclinical and clinical development of DNA topoisomerase I inhibitors in the United States. In: Andoh, T. (Ed.), Molecular Biology of DNA Topoisomerases and Its Application to Chemotherapy. International Symposium on DNA Topoisomerases in Chemotherapy. Boca Raton: CRC Press. pp. 301-311.

Rubin, E., Wood, V., Bharti, A., Trites, D., Lynch, C., and Kufe, D. (1994) A Phase I trial of 9-aminocamptothecin (9-AC). Proc. Am. Assoc. Cancer Res. 35, 245. (Abstract)

Takimoto, C.H, Klecker, R.W., Dahut, W.L., Yee, L.K., Strong, J.M., Allegra, C.J., and Grem, J.L. (1994) Analysis of the active lactone form of 9aminocamptothecin in plasma using solid-phase extraction and high-performance liquid chromatography. J. Chromatogr. B Biomed. Appl. 655, 97-104.

Takimoto, C.H., Dahut, W., Marino, M.T., Nakashima, H., Liang, M.D., Harold, N., Lieberman, R., Arbuck, S.G., Band, R.A., Chen, A.P., Hamilton, J.M., Cantilena, L.R., Allegra, C.J., and Grem, J.L. (1997) Pharmacodynamics and pharmacokinetics of a 72-hour infusion of 9-aminocamptothecin in adult cancer patients. J. Clin. Oncol. 15, 1492-1501. 